\title{
Clinical impact of type 2 diabetes mellitus on outcomes after one-lung ventilation during thoracic surgery: a retrospective cohort study
}

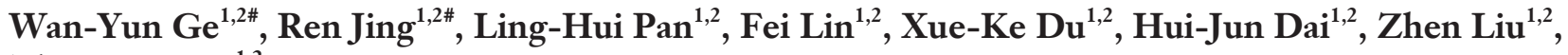 \\ Zhao-Kun $\mathrm{Hu}^{1,2} \wedge$
}

${ }^{1}$ Department of Anesthesiology, Guangxi Medical University Affiliated Cancer Hospital, Nanning, China; ${ }^{2}$ The Laboratory of Perioperative Medicine Research Center, Guangxi Medical University Affiliated Cancer Hospital, Nanning, China

Contributions: (I) Conception and design: ZK Hu; (II) Administrative support: LH Pan; (III) Provision of study materials or patients: WY Ge; (IV) Collection and assembly of data: WY Ge, R Jing; (V) Data analysis and interpretation: R Jing; (VI) Manuscript writing: All authors; (VII) Final approval of manuscript: All authors.

"These authors contributed equally to this work.

Correspondence to: Zhao-Kun Hu, MD. Department of Anesthesiology, Guangxi Medical University Affiliated Cancer Hospital, He Di Rd. No. 71, Nanning, China. Email: hu286353977@163.com.

Background: The preoperative presence of diabetes mellitus (DM) has been recently demonstrated to be a risk factor for adverse events after thoracic surgery. However, the specific effects of presence of DM preoperatively on thoracic surgery is not known. This study aimed to investigate the association between preoperative DM and clinical outcomes and the short-term survival rates after thoracic surgery.

Methods: In this retrospective, observational, and matched-pair analysis study, patients receiving thoracic surgery from a tertiary university hospital in 2 consecutive years were grouped as either type 2 DM (T2DM) or controlled within the first 24 hours after surgery. Multivariate Cox regression was conducted to investigate the impact of T2DM within the first 24 hours of admission on in-intensive care unit (ICU) and hospital survival.

Results: Among the included thoracic patients, 41 (8.4\%) had T2DM and 450 (91.6\%) did not have T2DM. In the single-factor analyses, T2DM patients were shown to have a higher preoperative white blood cells (WBCs) count, increased release of immunoglobulin A, complement C3 and C4, impaired kidney function with high level of urea, and low expression of alanine aminotransferase (ALT) and monoamine oxidase (MAO). In multivariate analyses, the preoperative urea level was associated with a low-grade risk of dying for the ICU survival time. In contrast, preoperative complement C3 level favored a positive contribution in-ICU survival. Besides the complement C3 level, immunoglobulin A level remained a positive contribution in regression models of hospital survival.

Conclusions: Pre-admission T2DM was not associated with an increased in-ICU and hospital mortality among patients with thoracic surgery. Furthermore, they were accompanied by impaired kidney function, activated inflammation and liver function.

Keywords: Diabetes mellitus (DM); one-lung ventilation (OLV); thoracic surgery; survival

Submitted Oct 13, 2019. Accepted for publication Jul 27, 2020.

doi: 10.21037/apm-19-367

View this article at: http://dx.doi.org/10.21037/apm-19-367

\footnotetext{
^ ORCID: 0000-0002-3379-6549.
} 


\section{Introduction}

Diabetes mellitus (DM) is a global health priority characterized by chronic hyperglycemia, metabolic disorder and multiple organ damage. These symptoms cause many acute and severe long-term complications such as diabetic ketoacidosis, hyperosmolar hyperglycemic state, stroke, and cardiovascular and chronic kidney disease $(1,2)$. The global prevalence of DM is expected to increase remarkably by the year 2030 (3). In China, the prevalence of DM has rapidly increased from $0.67 \%$ in 1980 to $10.4 \%$ in 2013 (4). The proportion of thoracic patients with DM is also growing because of the worldwide increase in diabetes. In some studies, pre-existing diabetes has been a risk factor of prognosis and postoperative complications in surgery patients (5-7), and has been associated with increased mortality in the surgical intensive care unit (ICU) (8). However, the association between DM and mortality among thoracic patients undergoing one-lung ventilation $(\mathrm{OLV})$ is still debatable.

Thoracic surgery of the heart, larger vessels, lung, esophagus, and mediastinum, often involves three major systems: the respiratory, circulatory and digestive systems. These systems especially influence the respiratory and circulatory functions such as hypoxic pulmonary vasoconstriction, paradoxical respiration, a decline of ventilation-perfusion ratio and cardiac output (9). Recent studies $(10,11)$ have reported that the lung is a primary organ that is impaired by DM. Therefore, thoracic patients with a pre-existing diabetes may have undesirable clinical outcomes and prognosis because of chronic hyperglycemia, metabolic disorder and multiple organ damage.

In order to validate the association between DM and mortality among thoracic patients undergoing OLV in an affiliated tumor hospital of university and to account for "real-life" conditions away from study protocols, this study investigated the impact of type $2 \mathrm{DM}$ (T2DM) on outcomes after OLV during thoracic surgery by means of a retrospective design. We present the following article in accordance with the STROBE reporting checklist (available at http://dx.doi.org/10.21037/apm-19-367).

\section{Methods}

\section{Patients}

This study was conducted in accordance with the Declaration of Helsinki (as revised in 2013). This retrospective study was approved by the institutional research ethics committee of the Guangxi Medical University Affiliated Cancer Hospital (No. LW2020020) and individual consent for this retrospective analysis was waived. Patients who underwent thoracic surgery at the Guangxi Medical University Affiliated Cancer Hospital were enrolled in this study $(\mathrm{n}=491)$. From July 2015 to June 2018, 944 patients underwent thoracic surgery at the Department of Thoracic Surgery of Affiliated Tumor Hospital of Guangxi Medical University. Among these patients, 309 patients who were missing complete data; 126 patients who were confirmed with pulmonary metastasis, intrapulmonary metastasis, invasion or recurrence; and 18 patients who received bronchial occlusion tube were excluded from the study. General and operation information for patients and laboratory blood test results was collected.

Standards of medical care for T2DM in China 2019 (4) was adopted before surgery for T2DM confirmation. There are three diagnostic criteria in this guideline: (I) typical diabetic symptoms including polydipsia, polyuria, polydipsia and weight loss plus random blood glucose test, showing a glucose level in venous plasma greater than or equal to $11.1 \mathrm{mmol} / \mathrm{L}$; (II) a fasting blood glucose test showing a glucose level in venous plasma greater than or equal to $7.0 \mathrm{mmol} / \mathrm{L}$; (III) a $2 \mathrm{~h}$ blood glucose test after glucose load showing a glucose level in venous plasma greater than or equal to $11.1 \mathrm{mmol} / \mathrm{L}$.

Clinical routine data were acquired from the 2electronic patient data management systems operated by the hospital (Yongyou Hospital Information System H6 V6.0, Beijing, China and DoCare Anesthesia Information System V5.0, Medicalsystem, Suzhou, China). The patients who met one or over more of the criteria were included into the observational group. The others were defined as the control group. We used propensity score matching analysis to minimize the selection bias between the observational and control groups. The propensity score matching was conducted using R 3.4.0 software. In this study, T2DM patients were matched to controls using American Society of Anesthesiologists (ASA) classification, basic diseases and style of tumor as matching criteria prior to multivariate analyses.

\section{Inclusion criteria}

The medical records of patients who underwent thoracic surgery were reviewed. The follow-up period ended 1 year 


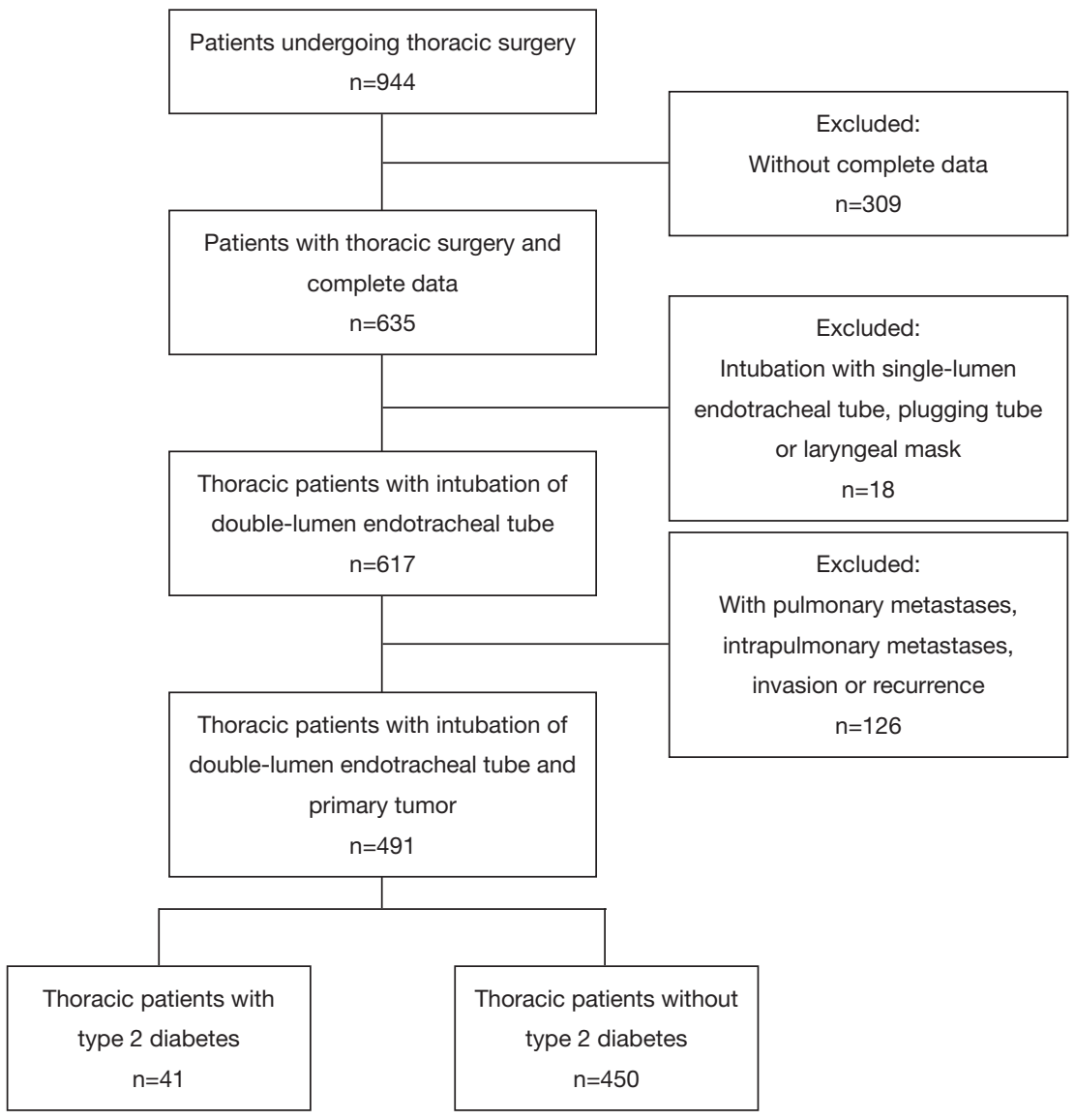

Figure 1 Flowchart of the study sample.

after patients' discharge from the hospital.

\section{Exclusion criteria}

The inclusion criteria were defined as patients aged $<18$ years; length of stay (LOS) in-ICU > 24 hours; undergoing reoperation or emergency operation; need for cardiac or pulmonary extracorporeal assist devices; and history of cardiovascular, liver, kidney or hematopoietic system disorders and psychiatric disease (see CONSORT diagram in Figure 1). Furthermore, patients with shorter observation intervals, which is shorter than 1 year of followup at the registry office, were excluded in analyses on longterm mortality.

\section{Statistical analyses}

Descriptive analyses and statistical testing were performed using the Stata version 11.0 (StataCorp, College Station,
TX, USA), with a $\mathrm{P}$ value below 0.05 being regarded as significant. When normal distribution was checked using the Shapiro Wilk test, results are given in mean \pm standard deviation, otherwise median and inter-quartile range are used. Qualitative variables are described by numbers with percent. Statistical significance among groups was analyzed by the $t$-tests, exact nonparametric Mann-Whitney $\mathrm{U}$ test or chi-square tests as appropriate. Survival was analyzed using Kaplan-Meier methods and evaluated by the log-rank test among groups. Multivariate testing was used to assess the impact of DM on hospital survival, and Cox-regression was adjusted for patient age, gender, body mass index (BMI), ASA classification, type of tumor as well as assessed variables with statistical difference. In this way, the proportion of DM patients and defined variables were compared over time. Furthermore, an alternative regression model that included year of admission as an explanatory variable was developed to investigate its impact on outcomes. All tests were defined as constituting explorative analysis as no 
adjustment was made for multiple testing.

\section{Results}

From the Department of Thoracic Surgery at the Guangxi Medical University Affiliated Cancer Hospital, 491 patients were enrolled in this study from July 2015 to June 2018. In total, the study included 291 male patients and 200 female patients. The mean age of these patients was 56.8 years. Among the included thoracic patients with double-lumen endotracheal tube intubation and primary tumor, 41 (8.4\%) had T2DM, while the 450 (91.6\%) who did not have $\mathrm{T} 2 \mathrm{DM}$ were set as the control group.

The baseline characteristics of all 491 patients who underwent thoracic surgery with double-lumen endotracheal tube intubation and primary tumors are shown in Table 1. The mean age was 61.4 years, and $65.9 \%$ of the thoracic patients with T2DM were male. The mean age of the thoracic patients with T2DM was significantly higher than that of patients without T2DM $(\mathrm{P}=0.016)$. Also, there were no statistical differences noted in the comparison of smoking history or ASA classification. However, the incidence of hypertension, arrhythmia and coronary heart disease (CHD) in the patients with T2DM were significantly increased compared with the control group.

Furthermore, the preoperative value of hemoglobin (HGB), total bilirubin (TBIL), albumin, alanine aminotransferase (ALT), lactate dehydrogenase (LDH), monoamine oxidase (MAO), and creatine kinase (CK) level were not significantly different between the groups. Compared with the patients without T2DM, the baseline value of white blood cells (WBCs), urea, immunoglobulin A, complement $\mathrm{C} 3$ and $\mathrm{C} 4$ in the patients with T2DM was significantly increased, while aspartate aminotransferase (AST) and glomerular filtration rate (GFR) value was decreased. In both unmatched and matched populations, the patients with T2DM showed higher WBC count, urea, IgA, C3 and C4, and decreased AST level (Table 1).

Time indexes such as duration of surgery, duration of anesthesia and duration of OLV were not significantly different from those for the patients with or without T2DM \{duration of surgery: 167 [107, 236] vs. 164 [121, 210], $\mathrm{P}=0.531$; duration of anesthesia: $210[150,264]$ vs. 205 [155, 257], $\mathrm{P}=0.624$; duration of OLV: 149 [91, 198] vs. 138 [96, 185], $\mathrm{P}=0.759$. It is evident that the mortality of the ICU and hospital in the patients with T2DM was significantly higher than that of the patients without T2DM (ICU: $7.3 \%$ vs. $0.2 \%, \mathrm{P}=0.002$, Figure 2 ; hospital: $12.2 \%$ vs. $0.2 \%$,
$\mathrm{P}=0.000$, Figure 3).

Next, blood transfusion, urine volume, blood loss volume, and type of surgery were similar between the groups (Table 2). There was no significant difference in the lung and mediastinum between the groups when comparing the location of the surgery. The proportion of esophagus surgery in the patients with $\mathrm{T} 2 \mathrm{DM}$ was lower than in the patients without T2DM (2.4\% vs. $14.2 \%, \mathrm{P}=0.033)$. The proportion of adenocarcinoma in the patients with T2DM was $58.5 \%(24 / 41)$, while it was $42.4 \%(191 / 450)$ in the control group $(\mathrm{P}=0.047)$. However, the proportion of squamous cell carcinoma, small cell lung cancer and other tumors was not different between the groups.

In the unmatched population, the patients with T2DM showed lower AST levels in comparison with those without T2DM ( $\mathrm{P}=0.037)$. Furthermore, the levels of albumin and LDH were lower in the patients with T2DM than those without T2DM in the matched population (albumin: $\mathrm{P}=0.040$; LDH: $\mathrm{P}=0.033$ ). In unmatched and matched population, the MAO level tended to be lower in the patients with T2DM (unmatched: $\mathrm{P}=0.000$; matched: $\mathrm{P}=0.001$ ) than in those without T2DM. However, the urea level was significantly increased in the patients with T2DM (unmatched: $\mathrm{P}=0.018$; matched: $\mathrm{P}=0.016$ ) than in the control group. It was also revealed that there were no differences in postoperative complications such as pulmonary infection, emphysema or bullae, atelectasis, pneumothorax, respiratory failure, and thrombosis between the groups (Table 3).

To account for remaining confounders after pair matching, we conducted Cox regression analysis for both the in-ICU and in-hospital groups, adjusting for patient age, gender, BMI, ASA classification, type of tumor, as well as assessed variables with a statistical difference. Notably, T2DM was not associated with an increased risk of dying in both ICU survival [hazard ratio (HR): $-0.131 ; 95 \%$ confidence interval (CI): -0.943 to $0.680 ; \mathrm{P}=0.751]$ and hospital survival (HR: 0.351; $95 \%$ CI: -0.455 to 1.157 ; $\mathrm{P}=0.393)$. Firstly, preoperative urea level was associated with a low-grade risk of dying in-ICU survival (HR: 0.024; $95 \%$ CI: 0.002 to $0.046 ; \mathrm{P}=0.036$ ). In contrast, the preoperative C3 level favored a positive contribution in-ICU survival (HR: $-1.926 ; 95 \%$ CI: -3.736 to $-0.116 ; \mathrm{P}=0.037$ ). Besides the $\mathrm{C} 3$ level, IgA level remained as a positive contribution in regression models of hospital survival (Table 4).

\section{Discussion}

In this observational, matched-pairs analysis study, we 
Table 1 Demographic and pre-operative characteristics of enrolled patients

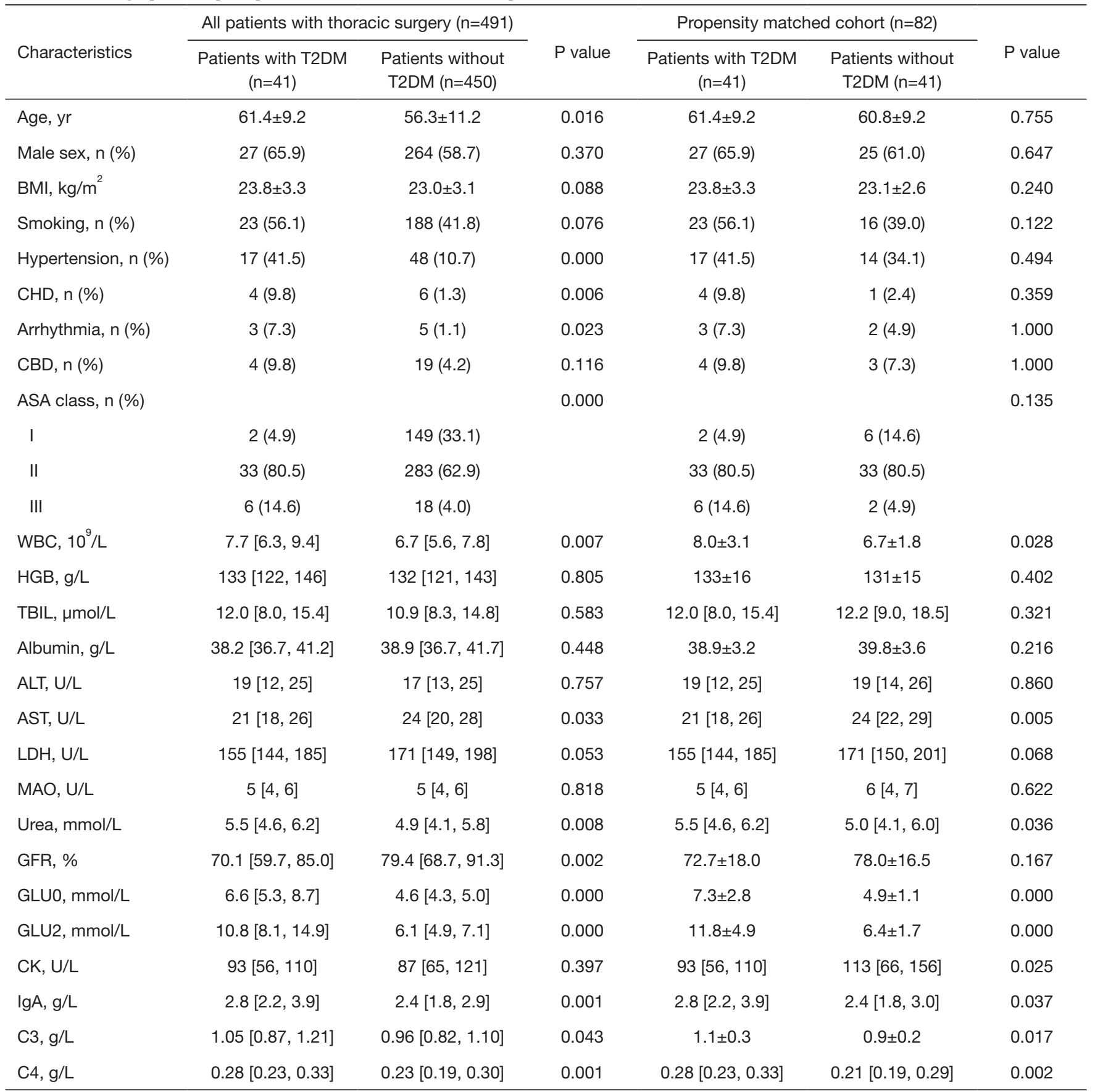

T2DM, type 2 diabetes mellitus; BMI, body mass index; CHD, coronary heart disease; CBD, cerebrovascular disease; ASA, American Society of Anesthesiologists; WBC, white blood cell; HGB, hemoglobin; TBIL, total bilirubin; ALT, alanine aminotransferase; AST, aspartate aminotransferase; LDH, lactate dehydrogenase; MAO, monoamine oxidase; GFR, glomerular filtration rate; GLU0, fasting blood glucose; GLU2, plasma glucose of 2 hours after meal; CK, creatine kinase; IgA, immunoglobulin A; C3, complement C3; C4, complement C4. 


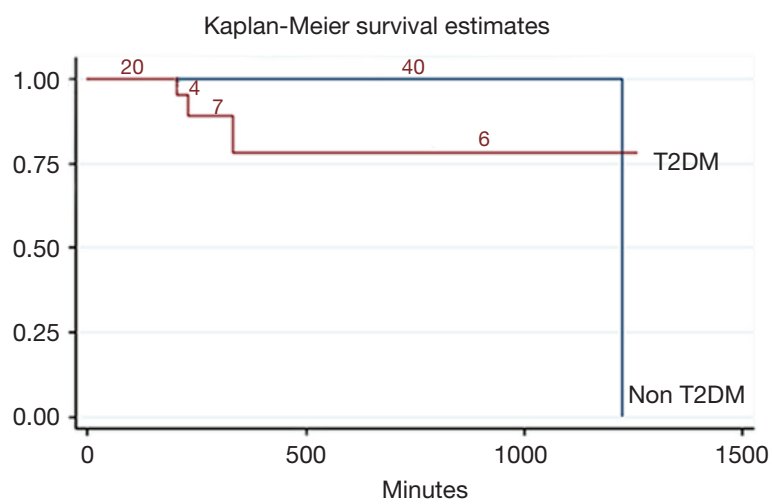

Figure 2 Kaplan-Meier curves for in-ICU survival in the matched cohort. ICU, intensive care unit; T2DM, type 2 diabetes mellitus.

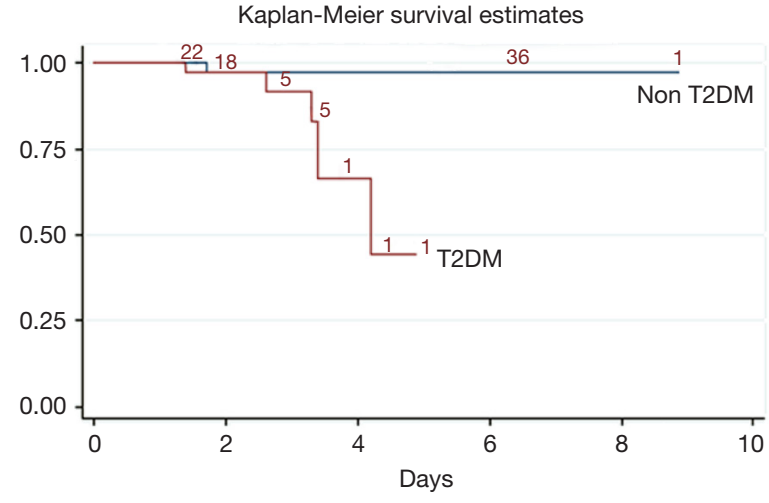

Figure 3 Kaplan-Meier curves for in-hospital survival in the matched cohort. T2DM, type 2 diabetes mellitus.

Table 2 Intraoperative parameters of enrolled patients

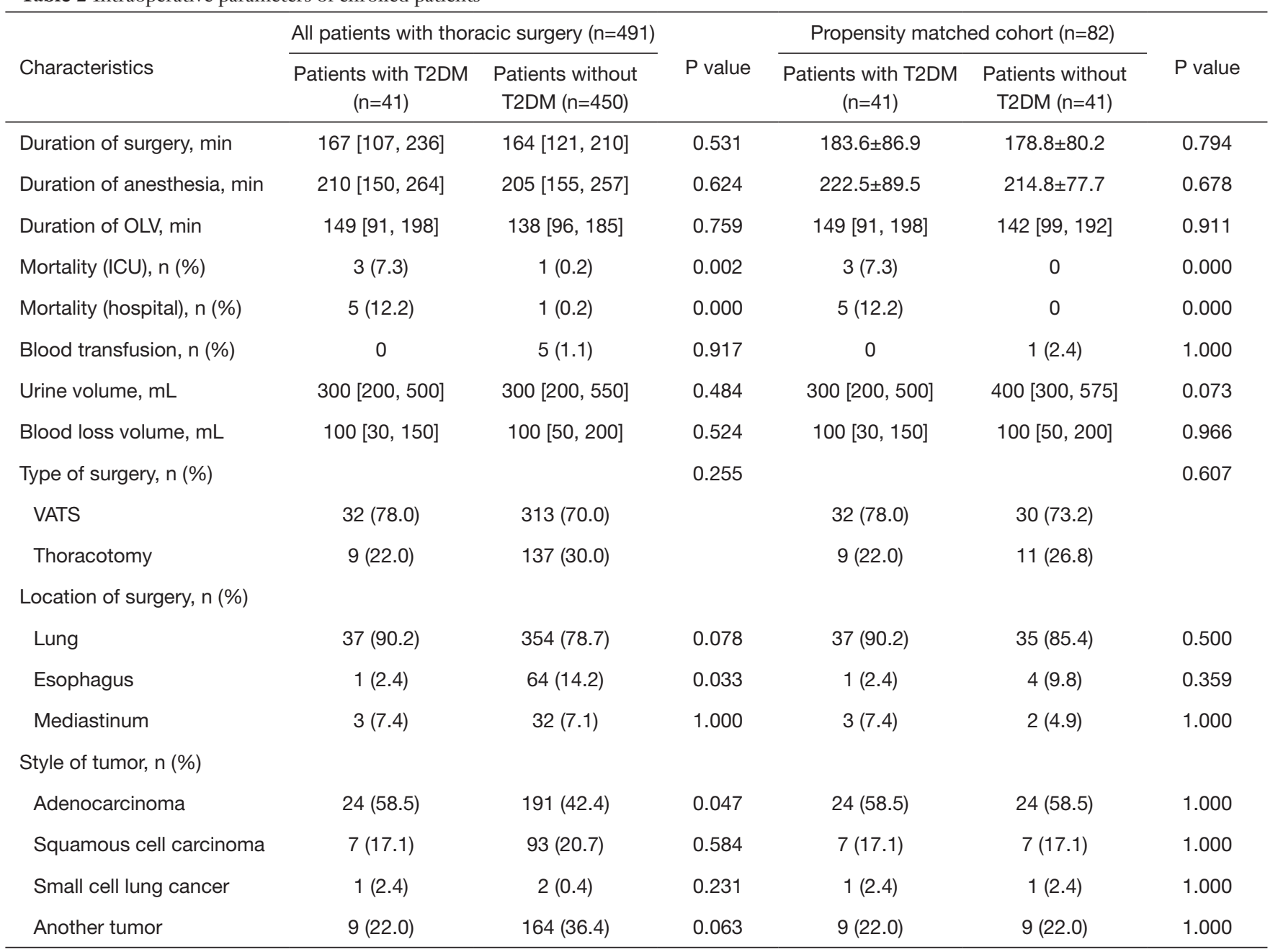

T2DM, type 2 diabetes mellitus; OLV, one-lung ventilation; ICU, intensive care unit; VATS, video-assisted thoracoscopic surgery. 
Table 3 Postoperative variables of enrolled patients

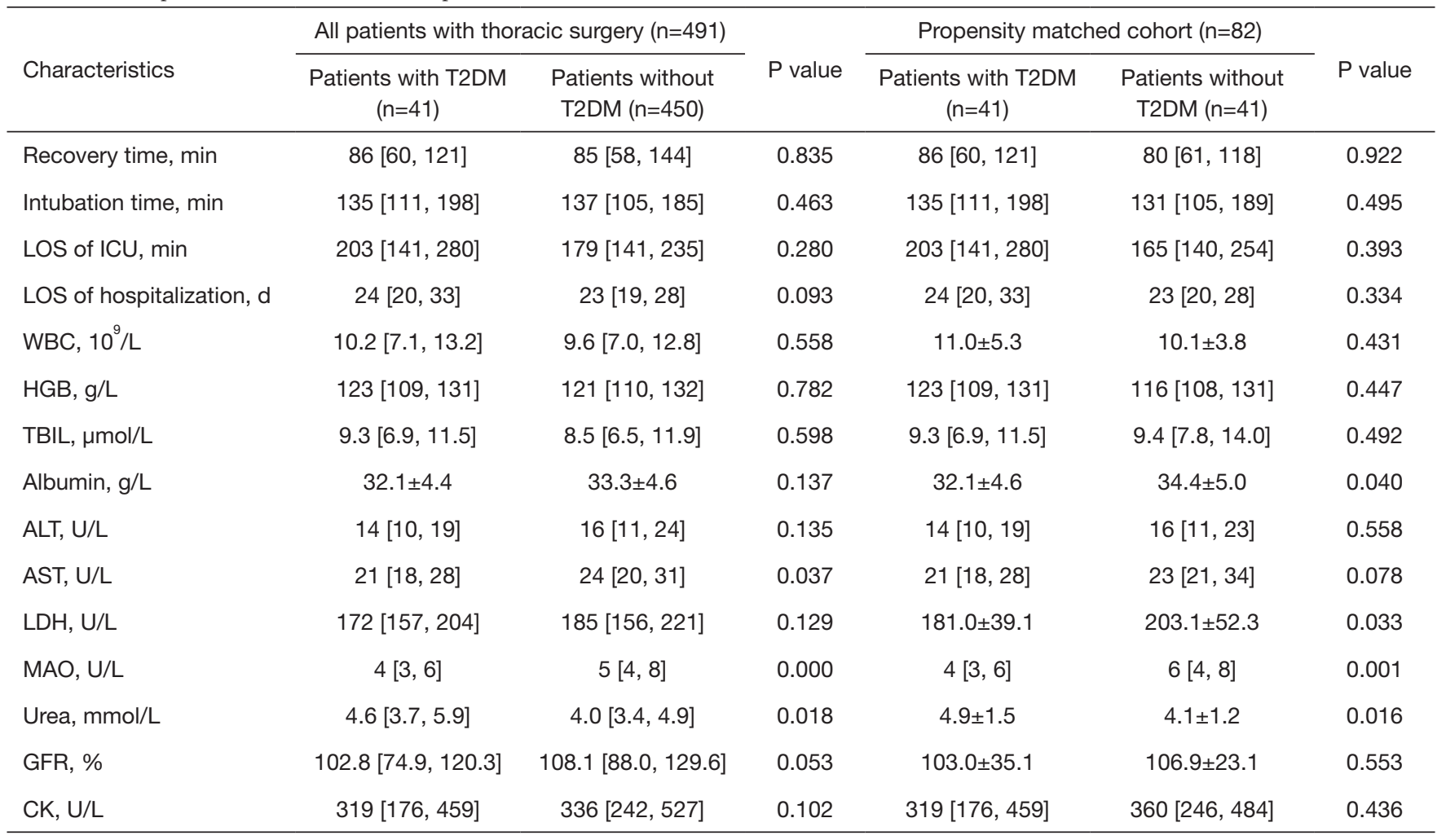

T2DM, type 2 diabetes mellitus; LOS, length of stay; ICU, intensive care unit; WBC, white blood cell; HGB, hemoglobin; TBIL, total bilirubin; ALT, alanine aminotransferase; AST, aspartate aminotransferase; LDH, lactate dehydrogenase; MAO, monoamine oxidase; GFR, glomerular filtration rate; $\mathrm{CK}$, creatine kinase.

showed that T2DM impairs clinical outcomes as it leads to an increase in-ICU and in-hospital mortality. In the singlefactor analyses, T2DM patients undergoing thoracic surgery were shown to have higher preoperative WBC count, increased release of $\mathrm{IgA}, \mathrm{C} 3$, and $\mathrm{C} 4$, impaired kidney functions with a high level of urea, and low expression of AST and MAO. In the multivariate analyses, preoperative urea level was associated with a low-grade risk of dying inICU survival. On the contrary, the preoperative $\mathrm{C} 3$ level favored a positive contribution in-ICU survival. Besides the C3 level, IgA level remained as a positive contribution in regression models of hospital survival.

WBC counts and fractions are associated with DM, and various components of metabolic syndrome and complications $(12,13)$. These findings are thought to be related to the activation of WBC by advanced glycation end-products and the production of pro-inflammatory cytokines (13-15). DM and its vascular complications are firmly related to inflammation and immune response. A study (16) showed that the incidence of bacterial infection in DM patients was 1.2 times higher than that if healthy volunteers, and the mortality of DM patients whose blood sugar was effectively controlled was more than 2.0 times higher than that of healthy volunteers. High blood sugar level would activate various protein kinase C isoforms, which regulate the expression of nitric oxide, endothelin, nuclear transcription factor-kB, and plasminogen activator inhibitor (PAI) to affect the release of cytokines and insulin $(17,18)$. This study also showed that thoracic patients with T2DM were accompanied by higher preoperative WBC count, suggesting activated inflammation.

High serum IgA level in DM patients is an early response to advanced glycosylation. Previous studies (19-21) have demonstrated that high serum IgA level correlates with assessments of blood sugar and almost $30 \%$ of DM patients have high serum IgA level. Plasma concentration of $\mathrm{C} 3$ is regarded as an independent risk marker for the incidence of DM; and may affect the level of blood sugar and insulin (22). Hess et al. (23) recently reported that plasma C3 levels, similarly to PAI-1, are independently associated 
Table 4 Cox regression analysis for in-ICU survival (left) and in-hospital survival (right)

\begin{tabular}{|c|c|c|c|c|c|c|}
\hline Characteristics & \multicolumn{3}{|c|}{ Cox regression for in-ICU survival } & \multicolumn{3}{|c|}{ Cox regression for in-hospital survival } \\
\hline T2DM & 0.751 & -0.131 & -0.943 to 0.680 & 0.393 & 0.351 & -0.455 to 1.157 \\
\hline Age, yr & 0.059 & 0.035 & -0.001 to 0.071 & 0.231 & 0.021 & -0.014 to 0.056 \\
\hline Male gender & 0.302 & 0.323 & -0.290 to 0.937 & 0.235 & -0.395 & -1.048 to 0.257 \\
\hline Hypertension & 0.494 & -0.219 & -0.848 to 0.409 & 0.660 & -0.138 & -0.752 to 0.476 \\
\hline $\mathrm{CHD}$ & 0.282 & 0.749 & -0.616 to 2.114 & 0.891 & -0.093 & -1.419 to 1.233 \\
\hline Arrhythmia & 0.346 & 0.731 & -0.789 to 2.251 & 0.951 & 0.042 & -1.280 to 1.363 \\
\hline ASA & 0.658 & 0.064 & -0.219 to 0.348 & 0.742 & 0.050 & -0.248 to 0.348 \\
\hline AST & 0.492 & 0.057 & -0.106 to 0.221 & 0.316 & 0.079 & -0.075 to 0.232 \\
\hline MAO & 0.204 & -0.196 & -0.499 to 0.107 & 0.622 & -0.066 & -0.328 to 0.196 \\
\hline Urea & 0.036 & 0.024 & 0.002 to 0.046 & 0.622 & 0.006 & -0.017 to 0.029 \\
\hline GFR & 0.906 & -0.010 & -0.173 to 0.153 & 0.501 & -0.078 & -0.304 to 0.149 \\
\hline GLUO & 0.472 & 0.039 & -0.067 to 0.144 & 0.709 & -0.022 & -0.139 to 0.094 \\
\hline GLU2 & 0.594 & -0.091 & -0.425 to 0.244 & 0.205 & 0.237 & -0.130 to 0.605 \\
\hline $\lg A$ & 0.093 & 1.448 & -0.240 to 3.137 & 0.044 & -1.737 & -3.426 to -0.047 \\
\hline C3 & 0.037 & -1.926 & -3.736 to -0.116 & 0.679 & -0.668 & -3.837 to 2.501 \\
\hline
\end{tabular}

ICU, intensive care unit; HR, ; Cl, ; T2DM, type 2 diabetes mellitus; BMI, body mass index; CHD, coronary heart disease; ASA, American Society of Anesthesiologists; WBC, white blood cell; AST, aspartate aminotransferase; MAO, monoamine oxidase; GFR, glomerular filtration rate; GLU0, fasting blood glucose; GLU2, plasma glucose of 2 hours after meal; IgA, immunoglobulin A; C3, complement C3; C4, complement C4.

with fibrin clot lysis in individuals with T2DM, suggesting C3 level would be regarded as a surrogate marker of fibrinolysis in T2DM patients. A nested case-control study in China showed that the risk of developing T2DM was still closely associated with elevated serum $\mathrm{C} 3$ levels [odds ratio (OR): $1.652,95 \% \mathrm{CI}: 1.004$ to $2.717, \mathrm{P}<0.05]$ (24). Therefore, plasma IgA and C3 were a protective response of activated inflammation in T2DM. In present study, T2DM patients undergoing thoracic surgery showed increased release of IgA, C3, and C4, but only IgA and C3 levels were positive protective factors of in-ICU and hospital survival.

The kidney is a common target organ of T2DM, and approximately $35 \%$ T2DM patients exhibit renal impairment (25). An increased plasma urea level is associated with acute and chronic renal dysfunction, heart failure, shock, and burns. The population with T2DM in the present study also had an increased level of urea, suggesting that kidney functions had varying degrees of impairment. However, impaired renal function did not aggravate the in-ICU and in-hospital survival rate, and 
thus requires further studies with larger sample sizes to investigate.

Except for the impairment of hepatocytes, the plasma levels of ALT and AST were simultaneously and significantly associated with T2DM incidence $(26,27)$ Previous studies (26-28) had reported that high ALT levels were independent risk factors for predicting T2DM, and the incidence of T2DM in patients with high ALT level was 1.27 times higher than those with low ALT levels. Interestingly, a difference for plasma ALT levels between the patients with T2DM and without T2DM was not observed in this study, while the plasma AST levels in T2DM patients was decreased in comparison with the patients without T2DM. AST is present in both hepatocyte cytoplasm and mitochondria, and is an essential metabolic link between carbohydrate and protein metabolism (29). However, determining the relationship between plasma AST level sand T2DM in thoracic surgery should perform various further research in the future.

Overall, it appears when undergoing thoracic surgery, patients with T2DM are accompanied by low expression of MAO than the patients without T2DM, and no published data can explain this phenomenon comprehensively. It could perhaps be explained by the blood sugar stability and endothelial-dependent relaxation since the T2DM patients received higher amounts of insulin and hypoglycemic drugs. A recent study suggested that MAO-A is induced in diabetic aortas, and vitamin $\mathrm{D}$ improves diabetes-induced endothelial dysfunction by modulating MAO-A expression (30). Exposure of high glucose and pro-inflammatory stimuli to cardiomyocytes causes $\mathrm{MAO}$-dependent increase in reactive oxygen species that leads to permeable transition pore opening and mitochondrial dysfunction; occurs upstream of endoplasmic reticulum stress, and is abolished by the MAO inhibitor, pargyline. Moreover, MAO inhibition has been shown to prevent both mast cell degranulation and altered collagen deposition, thereby normalizing diastolic function (31). Regardless of the presence of DM, in vitro inhibition of MAO significantly improves endothelialdependent relaxation in human mammary arteries, and MAO inhibitors are useful in restoring endothelial response to increased oxidative stress in patients with coronary artery disease and DM $(32,33)$.

In the present study, we first examined the effects of T2DM during thoracic surgery with OLV on clinical outcomes and short-term survival. Although the outcomes of surgery and anesthesia were similar, the inflammation and immune response in these patients with T2DM were significantly activated to adapt to high glucose and metabolic dysfunction. Furthermore, effective control of blood sugar decreased the endothelial response to increased oxidative stress and prevent mast cell degranulation.

Several limitations must be considered in the interpretation of our results. First, patients with T2DM, especially those undergoing thoracic surgery, might have been introduced for medical reasons, thus limiting the generalizability of the findings. However, this aspect was mitigated by excluding patients that could have had the above indications. Second, the quality of data that was considered in this retrospective analysis might have been inferior to that of prospective trials. On the other hand, the use of retrospective data is apt in creating a realistic picture of clinical routine, which may be essential to validate the findings of prospective trials. Lastly, the lack of large sample and long-term survival data restrict the reliability of this retrospective study. However, standard pre-operating procedures for T2DM were introduced before the start of the study period. Including the patient's admission year in an alternative multivariate regression analysis did not significantly affect the previously calculated HRs.

\section{Conclusions}

In summary, T2DM was found to have no significantly adverse effect on clinical outcomes of thoracic surgery in this study. However, considering that the definite effect of T2DM on the perioperative period of thoracic surgery was not reported in previous experimental studies, further large studies are needed to evaluate the non-antidiabetic effects of high glucose-induced inflammation on kidney and liver function for thoracic surgery.

\section{Acknowledgments}

We thank all co-authors for support from data collecting to revising manuscript.

Funding: The present study was supported by the National Natural Science Foundation of China (81460016).

\section{Footnote}

Reporting Checklist: The authors have completed the STROBE reporting checklist. Available at http://dx.doi. org/10.21037/apm-19-367

Data Sharing Statement: Available at http://dx.doi. 
org/10.21037/apm-19-367

Conflicts of Interest: All authors have completed the ICMJE uniform disclosure form (available at http://dx.doi. org/10.21037/apm-19-367). The authors have no conflicts of interest to declare.

Ethical Statement: The authors are accountable for all aspects of the work in ensuring that questions related to the accuracy or integrity of any part of the work are appropriately investigated and resolved. This study was conducted in accordance with the Declaration of Helsinki (as revised in 2013). This study was approved by the institutional research ethics committee of the Guangxi Medical University Affiliated Cancer Hospital (No. LW2020020) and individual consent for this retrospective analysis was waived.

Open Access Statement: This is an Open Access article distributed in accordance with the Creative Commons Attribution-NonCommercial-NoDerivs 4.0 International License (CC BY-NC-ND 4.0), which permits the noncommercial replication and distribution of the article with the strict proviso that no changes or edits are made and the original work is properly cited (including links to both the formal publication through the relevant DOI and the license). See: https://creativecommons.org/licenses/by-nc-nd/4.0/.

\section{References}

1. Hörber S, Achenbach P, Schleicher E. Harmonization of immunoassays for biomarkers in diabetes mellitus. Biotechnol Adv 2020;39:107359.

2. Pearson-Stuttard J, Blundell S, Harris T, et al. Diabetes and infection: assessing the association with glycaemic control in population-based studies. Lancet Diabetes Endocrinol 2016;4:148-58.

3. Khan MAB, Hashim MJ, King JK, et al. Epidemiology of type 2 diabetes - global burden of disease and forecasted trends. J Epidemiol Glob Health 2020;10:107-11.

4. Jia W, Weng J, Zhu D, et al. Standards of medical care for type 2 diabetes in China 2019. Diabetes Metab Res Rev 2019;35:e3158.

5. Greco G, Kirkwood KA, Gelijns AC, et al. Diabetes is associated with reduced stress hyperlactatemia in cardiac surgery. Diabetes Care 2018;41:469-77.

6. Yoshioka D, Toda K, Yokoyama JY, et al. Diabetes mellitus adversely affects mortality and recurrence after valve surgery for infective endocarditis. J Thorac Cardiovasc Surg 2018;155:1021-9.e5.

7. Garg R, Schuman B, Bader A, et al. Effect of preoperative diabetes management on glycemic control and clinical outcomes after elective surgery. Ann Surg 2018;267:858-62.

8. Ali Abdelhamid Y, Plummer MP, Finnis ME, et al. Longterm mortality of critically ill patients with diabetes who survive admission to the intensive care unit. Crit Care Resusc 2017;19:303-9.

9. Petrella F, Spaggiari L. The smaller the better: a new concept in thoracic surgery? Lancet Oncol 2016;17:699-700.

10. Klein OL, Krishnan JA, Glick S, et al. Systematic review of the association between lung function and type 2 diabetes mellitus. Diabet Med 2010;27:977-87.

11. Sonoda N, Morimoto A, Tatsumi Y, et al. A prospective study of the impact of diabetes mellitus on restrictive and obstructive lung function impairment: the Saku study. Metabolism 2018;82:58-64.

12. Mahdiani A, Kheirandish M, Bonakdaran S. Correlation between white blood cell count and insulin resistance in type 2 diabetes. Curr Diabetes Rev 2019;15:62-6.

13. Wheelock KM, Saulnier PJ, Tanamas SK, et al. White blood cell fractions correlate with lesions of diabetic kidney disease and predict loss of kidney function in type 2 diabetes. Nephrol Dial Transplant 2018;33:1001-9.

14. Al Rifai M, Schneider AL, Alonso A, et al. sRAGE, inflammation, and risk of atrial fibrillation: results from the Atherosclerosis Risk in Communities (ARIC) Study. J Diabetes Complications 2015;29:180-5.

15. Bower JK, Pankow JS, Lazo M, et al. Three-year variability in plasma concentrations of the soluble receptor for advanced glycation end products (sRAGE). Clin Biochem 2014;47:132-4.

16. Koziel H, Koziel MJ. Pulmonary complications of diabetes mellitus. Pneumonia. Infect Dis Clin North Am 1995;9:65-96.

17. Wang B, Yee Aw T, Stokes KY. N-acetylcysteine attenuates systemic platelet activation and cerebral vessel thrombosis in diabetes. Redox Biol 2018;14:218-28.

18. Ci LY, Liu DS, Yang JQ, et al. Expression of long non coding RNA and mRNA in the hippocampus of mice with type 2 diabetes. Mol Med Rep 2018;18:4960-8.

19. Bonifacio E, Ziegler AG, Klingensmith G, et al. Effects of high-dose oral insulin on immune responses in children at high risk for type 1 diabetes: the Pre-POINT randomized clinical trial. JAMA 2015;313:1541-9.

20. Naseri R, Mozaffari HR, Ramezani M, et al. Effect of diabetes mellitus type 2 on salivary glucose, 
immunoglobulin A, total protein, and amylase levels in adults: a systematic review and meta-analysis of casecontrol studies. J Res Med Sci 2018;23:89.

21. Kheirmand Parizi M, Akbari H, Malek-Mohamadi M, et al. Association of salivary levels of immunoglobulin-a and amylase with oral-dental manifestations in patients with controlled and non-controlled type 2 diabetes. BMC Oral Health 2019;19:175.

22. Ursini F, D Angelo S, Russo E, et al. Serum complement C3 and type 2 diabetes in rheumatoid arthritis: a casecontrol study. Rev Recent Clin Trials 2018;13:215-21.

23. Hess K, Alzahrani SH, Price JF, et al. Hypofibrinolysis in type 2 diabetes: the role of the inflammatory pathway and complement C3. Diabetologia 2014;57:1737-41.

24. Zhou H, Zhang Y, Zhang YH, et al. Predictive effects of serum complement factor 3 on type 2 diabetes mellitus: a nested case- control study. Chin J Diabetes Mellitus 2015;7:230-34.

25. Thomas MC, Cooper ME, Zimmet P. Changing epidemiology of type 2 diabetes mellitus and associated chronic kidney disease. Nat Rev Nephrol 2016;12:73-81.

26. Kaneko K, Yatsuya H, Li Y, et al. Association of gammaglutamyl transferase and alanine aminotransferase with type 2 diabetes mellitus incidence in middle-aged Japanese men: 12-year follow up. J Diabetes Investig 2019;10:837-45.

27. De Silva NMG, Borges MC, Hingorani AD, et al. Liver

Cite this article as: Ge WY, Jing R, Pan LH, Lin F, Du XK, Dai HJ, Liu Z, Hu ZK. Clinical impact of type 2 diabetes mellitus on outcomes after one-lung ventilation during thoracic surgery: a retrospective cohort study. Ann Palliat Med 2020;9(5):2455-2465. doi: 10.21037/apm-19-367 function and risk of type 2 diabetes: bidirectional mendelian randomization study. Diabetes 2019;68:1681-91.

28. Zhang J, Cheng N, Ma Y, et al. Liver enzymes, fatty liver and type 2 diabetes mellitus in a jinchang cohort: a prospective study in adults. Can J Diabetes 2018;42:652-8.

29. Sookoian S, Pirola CJ. Liver enzymes, metabolomics and genome-wide association studies: from systems biology to the personalized medicine. World J Gastroenterol 2015;21:711-25.

30. Sturza A, Văduva A, Uțu D, et al. Vitamin D improves vascular function and decreases monoamine oxidase $\mathrm{A}$ expression in experimental diabetes. Mol Cell Biochem 2019;453:33-40.

31. Deshwal S, Forkink M, Hu CH, et al. Monoamine oxidase-dependent endoplasmic reticulum-mitochondria dysfunction and mast cell degranulation lead to adverse cardiac remodeling in diabetes. Cell Death Differ 2018;25:1671-85.

32. Lighezan R, Sturza A, Duicu OM, et al. Monoamine oxidase inhibition improves vascular function in mammary arteries from nondiabetic and diabetic patients with coronary heart disease. Can J Physiol Pharmacol 2016;94:1040-7.

33. Duicu OM, Lighezan R, Sturza A, et al. Assessment of mitochondrial dysfunction and monoamine oxidase contribution to oxidative stress in human diabetic hearts. Oxid Med Cell Longev 2016;2016:8470394. 